'Departamento de

Endocrinología, Escuela de Medicina, Pontificia Universidad Católica de Chile. Santiago, Chile.

${ }^{2}$ Departamento de Anatomía Patológica, Escuela de Medicina, Pontificia Universidad Católica de Chile. Santiago, Chile.

${ }^{3}$ Servicio de Anatomía Patológica, Hospital Naval A. Nef. Viña de Mar, Chile.

${ }^{4}$ Departamento de Patología y Fisiología, Escuela de Medicina, Universidad de Valparaíso. Viña del Mar, Chile.

${ }^{5}$ Servicio de Medicina, Hospital Naval A. Nef. Viña del Mar, Chile.

${ }^{6}$ Departamento de Medicina, Escuela de Medicina, Universidad de Valparaíso. Viña del Mar, Chile.

Trabajo no recibió financiamiento. Los autores declaran no tener conflictos de interés.

Recibido el 18 de julio de 2018 aceptado el 13 de noviembre de 2018.

Correspondencia a: Jorge Vega

Dirección 5 Norte 1035, Viña del Mar, Chile. jvegastieb@gmail.com

\section{Hipofisitis linfoplasmocitaria y fibrosis retroperitoneal asociadas a un síndrome poliglandular autoinmune. Enfermedades relacionadas con IgG4. Caso clínico}

\author{
FRANCISCO J. GUARDA ${ }^{1}$, GONZALO P. MÉNDEZ², \\ ROBERTO ESPINOSA ${ }^{3,4}$, ROGER GEJMAN ${ }^{2}$, JORGE VEGA ${ }^{5,6}$
}

\section{Hypophysitis and retroperitoneal fibrosis associated with autoimmune polyglandular syndrome and IgG4-related disease. Report of one case}

We report a 23 year old woman presenting with a nephrotic syndrome due to minimal change disease, central diabetes insipidus, primary hypothyroidism, vitiligo and universal alopecia. Eleven years later, she presented secondary amenorrhea due to hypogonadotropic hypogonadism, with mild hyperprolactinemia and central adrenal insufficiency. A magnetic resonance imaging of the sella turcica showed a pituitary mass with suprasellar extension that was resected using a transsphenoidal approach. Pathology confirmed the presence of a lymphoplasmacytic hypophysitis. She needed a second surgical resection due to mass growth and neuro-ophthalmologic impairment. One year later, systemic lupus erythematosus, arterial hypertension and type 2 diabetes mellitus were diagnosed. Two years later, due to back pain, constipation and renal failure, retroperitoneal fibrosis was found, satisfactorily treated with glucocorticoids and colchicine. Hence, this clinical vignette shows the coexistence of autoimmune polyglandular syndrome with retroperitoneal fibrosis and lymphoplasmacytic hypophysitis. Tissue analysis showed the presence of IgG4 producing plasma cells in the pituitary and retroperitoneum, which constitute a basis for the diagnosis of IgG4 related disease.

(Rev Med Chile 2018; 146: 1486-1492)

Key words: Autoimmune; Autoimmune Hypophysitis; Hypothyroidism; Polyendocrinopathies; Retroperitoneal Fibrosis; Immunoglobulin G4-Related Disease.

\section{L} a hipofisitis es una patología infrecuente. Histológicamente puede clasificarse en tica, granulomatosa, xantomatosa, ente, plasmocítica y mixta. Puede ser primaria o secundaria a granulomatosis con poliangeitis, tuberculosis, sarcoidosis, sífilis, histiocitosis, tumores selares y drogas inmunomoduladoras, entre otras ${ }^{1-4}$. La hipofisitis linfoplasmocitaria (HLP) ha sido descrita entre las múltiples manifestaciones de la enfermedad relacionada con IgG4 (IgG4-RD) 5

La IgG4-RD corresponde a una entidad cada día más reconocida en la práctica clínica, caracterizada por compromiso fibroinflamatorio 
linfoplasmocitario de diversos tejidos. Múltiples criterios han sido postulados para el diagnóstico de HLP secundaria a IgG4, siendo la combinación de éstos y de características clínicas los que le dan la especificidad. Dentro de estos se encuentran la histopatología hipofisaria concordante con infiltrado mononuclear IgG4 positivo, resonancia magnética compatible, biopsia de otros tejidos concordante, IgG4 sérica elevada y respuesta clínica a corticoides sistémicos ${ }^{2}$.

La fibrosis retroperitoneal (FRP) es también una patología infrecuente caracterizada por la presencia de tejido fibroinflamatorio en el retroperitoneo. Clásicamente puede comprometer aorta, vena cava inferior y uréteres, originando insuficiencia renal obstructiva. En la mayoría de los casos es idiopática, pero puede ser secundaria a drogas, enfermedades autoinmunes, desórdenes fibrosantes, exposición a asbesto, IgG4-RD, cirugía, radioterapia, quimioterapia y traumatismos ${ }^{6,7}$.

El síndrome poliglandular autoinmune (APS) es un grupo de condiciones inmunomediadas que afecta órganos endocrinos y no-endocrinos. Cuatro subtipos han sido descritos, siendo más común el tipo 2 (síndrome de Schmidt) con una prevalencia de 1-2 casos por 100.000 individuos. Afecta principalmente a mujeres jóvenes y se ha definido como la presencia de insuficiencia adrenal primaria (o evidencia serológica de adrenalitis), asociada a diabetes mellitus $1(28-52 \%)$ y enfermedad tiroidea autoinmune (ETA) (70-83\%). Otras condiciones que pueden coexistir son hipogonadismo hipergonadotrófico (3-10\%), vitíligo (4-11\%), anemia perniciosa (1-25\%) y alopecia (2-5\%). Algunos autores han definido "APS tipo 3" cuando no existe insuficiencia adrenal primaria. "APS tipo 4" se diagnostica cuando no se cumplen los criterios de las anteriores, pero varios la consideran una variante de "APS tipo 2". El APS tipo 1 se presenta principalmente en niños y se caracteriza por candidiasis mucocutánea, hipoparatiroidismo primario y $\mathrm{ETA}^{8-11}$.

Comunicamos el caso de una mujer con HLP en asociación con APS, que presentó varios años después una FRP, diagnosticándose una enfermedad relacionada con IgG4.

\section{Caso clínico}

Mujer de 23 años, asmática, con desarrollo puberal normal y ciclos menstruales regulares, que consultó por un síndrome nefrótico y exantema vesicular en áreas foto-expuestas. Su función renal era normal y la serología para lupus eritematoso sistémico (LES) negativa. La biopsia renal mostró una nefrosis lipoídea. Se trató con prednisona con remisión completa. En los 3 años siguientes tuvo varias reactivaciones permaneciendo con corticoides 8 años.

Cuatro años después desarrolló poliuria. Aunque sus padres tenían diabetes mellitus 2 (DM2) diagnosticada antes de los 50 años, su glicemia pre y post-carga eran normales. Se efectuó un test de restricción acuosa que mostró elevación de la natremia y persistencia de la poliuria, normalizándose al administrar desmopresina. Un scanner de silla turca no mostró anormalidades. Se diagnosticó diabetes insípida (DI) y se prescribió desmopresina diaria.

Un año más tarde presentó intolerancia al frio, sequedad de piel y aumento de peso, sin bocio palpable. El laboratorio mostró TSH: > $100 \mathrm{uUI} /$ mL (VN: 0,8-4,4), T4: 1,9 ug/dL T3: 80,7 ng/mL (VN: 52-175), anticuerpos-antiperoxidasas: 36 $\mathrm{UI} / \mathrm{mL}(\mathrm{VN}:<12)$ y anticuerpos-antitiroglobulina: $233 \mathrm{UI} / \mathrm{mL}$ (VN: 10-60). Se prescribió levotiroxina $100 \mathrm{ug} /$ día mejorando sus síntomas y normalizando la TSH. Dos años después la DI desapareció. Se prescribió anticonceptivos hormonales combinados.

Un año después apareció una placa de alopecia, la cual se extendió constituyendo alopecia universal y apareció vitíligo que se generalizó. Meses después el pelo reapareció, pero totalmente blanco, dando la apariencia de albinismo. El cortisol sérico fue: 13,2 ug/dL (VN: 7-11) a las 08:00 AM; TSH: 0,93 uUI/mL, T4: 8,4 ug/dL (sin cambios en la dosis de tiroxina). No se efectuó test de estimulación con ACTH.

A los 34 años (11 años después del síndrome nefrótico), notó amenorrea al dejar los anticonceptivos. Existía un patrón de hipogonadismo hipogonadotrópico: FSH 0,8 mUI/mL (VN: 2,510), LH: $0,52 \mathrm{mUI} / \mathrm{mL}$ (VN: 1,9-2,5) con niveles indetectables de estradiol. La prolactina estaba elevada $(53,6 \mathrm{ng} / \mathrm{mL})$ (VN: 2,1-25). No se realizaron imágenes selares y se prescribió cabergolina empíricamente, normalizando los niveles de prolactina sin que reaparecieran las menstruaciones.

Un año más tarde los niveles de cortisol AM fueron bajos $(5,6 \mathrm{ug} / \mathrm{dL})$ con ACTH normalbaja (15,2 pg/mL, VN: 10-60), concordantes con 
insuficiencia adrenal central. No se efectuó test de estimulación con ACTH y se suplementó hidrocortisona en dosis fisiológicas. La RM de silla turca mostró aumento de volumen hipofisiario informado como macroadenoma con extensión supraselar (Figura 1). El campo visual fue normal y no presentaba síntomas de DI.

Debido al crecimiento progresivo de la hipófisis e inminente compromiso visual se efectuó resección transesfenoidal. La biopsia mostró una HLP con infiltración policlonal de células plasmáticas. Se prescribió prednisona, pero por progresión de la infiltración hipofisiaria y deterioro del campo visual, a pesar del tratamiento, una nueva resección transesfenoidal fue realizada. La histología fue similar a la descrita previamente (Figura 2), pero la inmunohistoquímica efectuada posteriormente mostró la presencia de células plasmáticas positivas para IgG4 (Figura 3). En el postoperatorio reapareció la DI prescribiéndose desmopresina. Con el fin de prevenir el crecimiento hipofisario, se efectuó radioterapia externa. Más tarde apareció hipolacrimia que se trató con lágrimas artificiales.

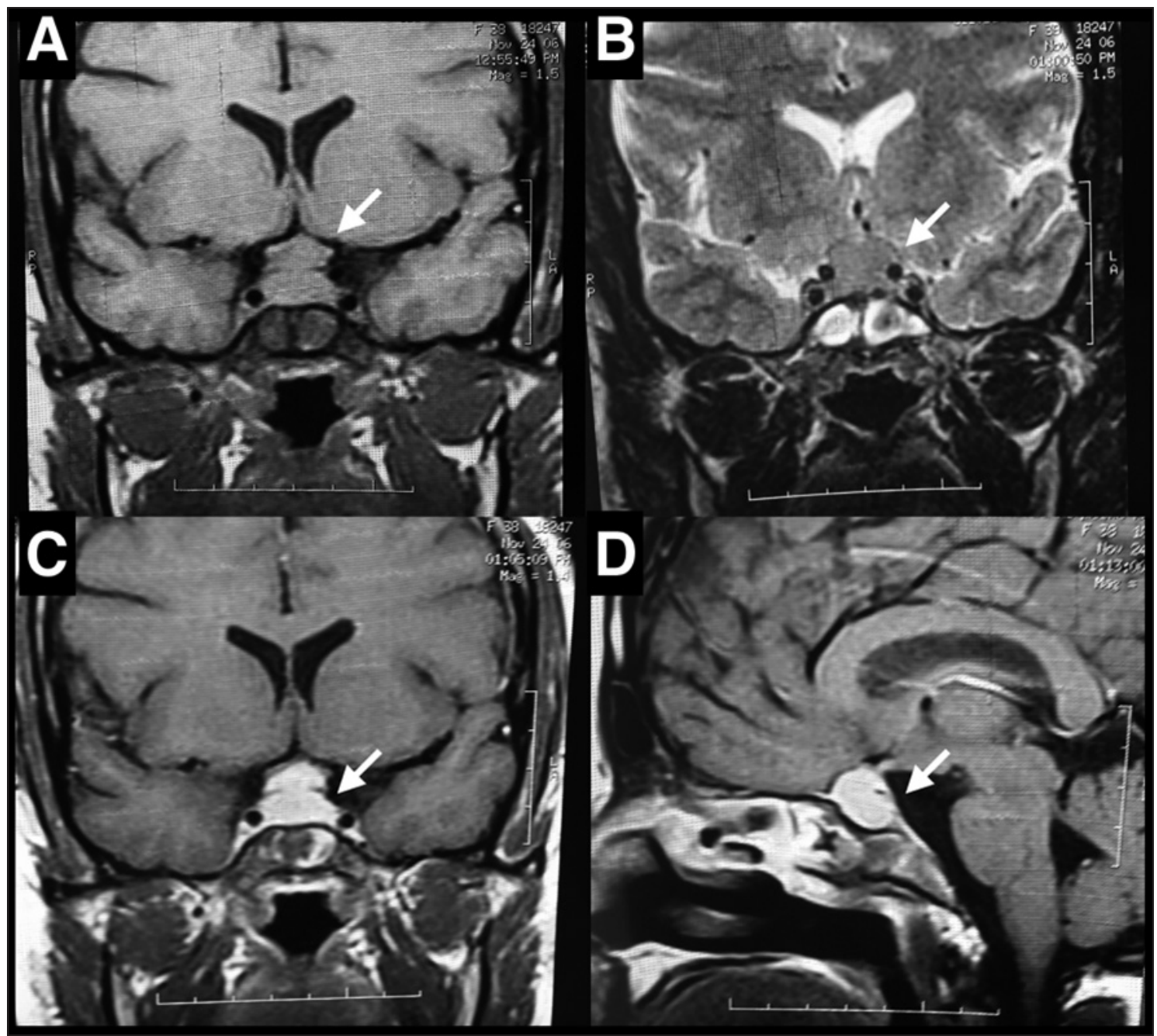

Figura 1. Resonancia magnética de silla turca. Imágenes coronales T1 (A) y T2 (B) sin contraste que muestran una masa selar isointensa con extensión supraselar y compromiso quiasmático. Imagen coronal T1 post-contraste (C) que muestra una lesión altamente contrastada, también visible en vista sagital (D) compatible con un proceso inflamatorio. Flechas blancas indican la lesión selar. 

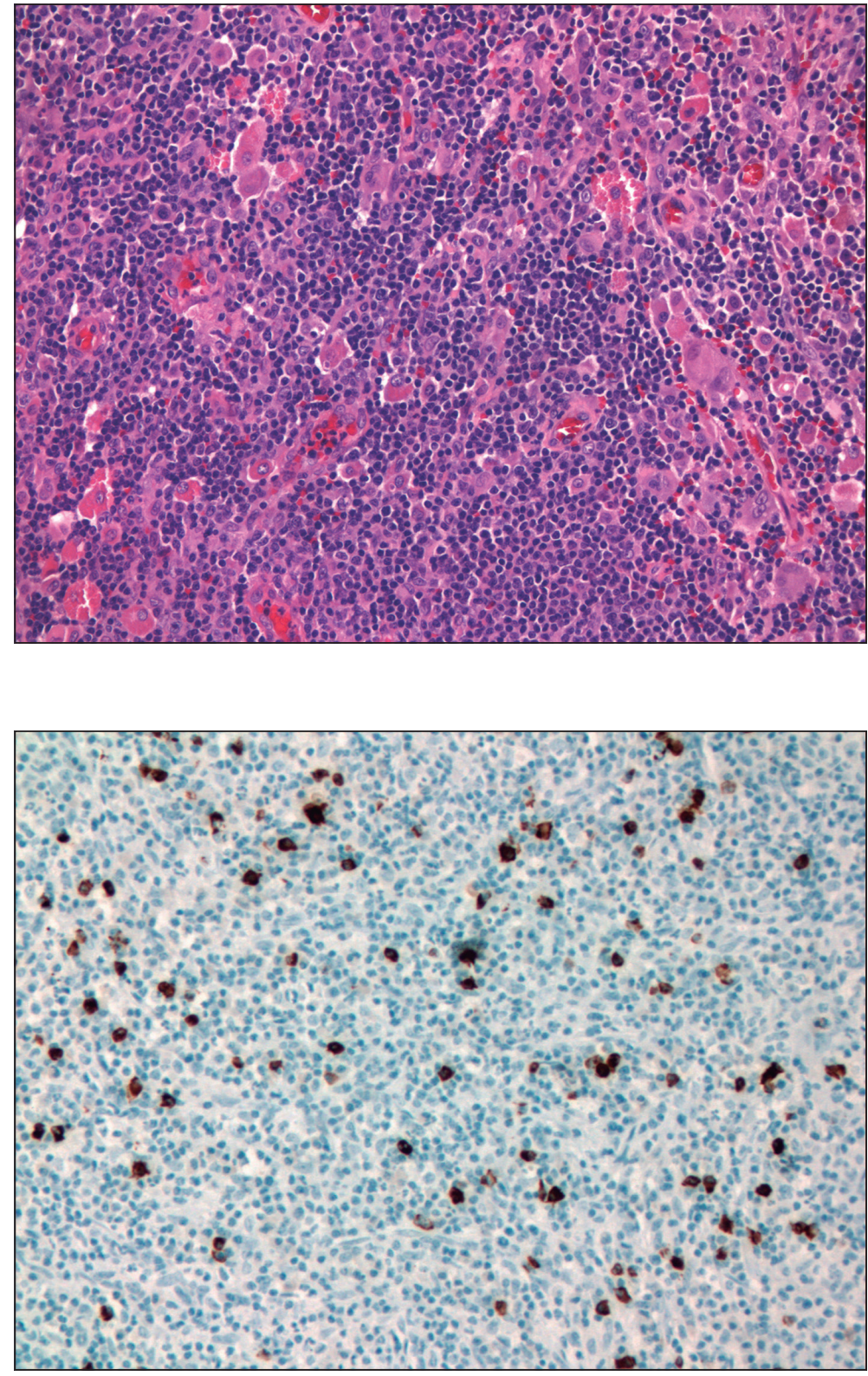

Figura 2. En el área pituitaria se observa un infiltrado intersticial difuso con predominio de células plasmáticas. Las células acidófilas y basófilas están aisladas o en pequeños grupos, como resultado de un intenso infiltrado inflamatorio que reemplaza y distorsiona la arquitectura pituitaria (hematoxilina y eosina; 200x).

Figura 3. Área pituitaria que muestra infiltrado inflamatorio con reactividad para IgG4 citoplasmática multifocal para células plasmáticas (Tinción inmunohistoquímica con anticuerpos monoclonales anti $\lg G 4 ; 200 x)$.

Un año después, en un control ambulatorio, se observó rash malar, eczema cutáneo en áreas fotoexpuestas y elevación de la VHS a $102 \mathrm{~mm} / \mathrm{h}$. Se sospechó LES y la serología confirmó el diagnóstico. Los anticuerpos antinucleares fueron $(+)$ 1: 1.280, C'3 fue bajo con C'4 normal y los anticuerpos anti-ENA, anti-DNA y anti-fosfolípidos fueron (-). La función renal y examen de orina fueron normales. Unos meses después se diag- nosticó hipertensión arterial y diabetes mellitus 2, prescribiéndose losartan y metformina.

Dos años más tarde presentó dolor lumbar e hipogástrico sordo, constipación, anemia y elevación de la creatinina a $1,4 \mathrm{mg} / \mathrm{dL}$. Un scanner mostró tejido peri-aórtico y peri-renal con hidronefrosis bilateral sugerente de FRP. La biopsia confirmó el diagnóstico. Se instalaron catéteres de pigtail y se prescribió $30 \mathrm{mg}$ de prednisona y $0,5 \mathrm{mg}$ 

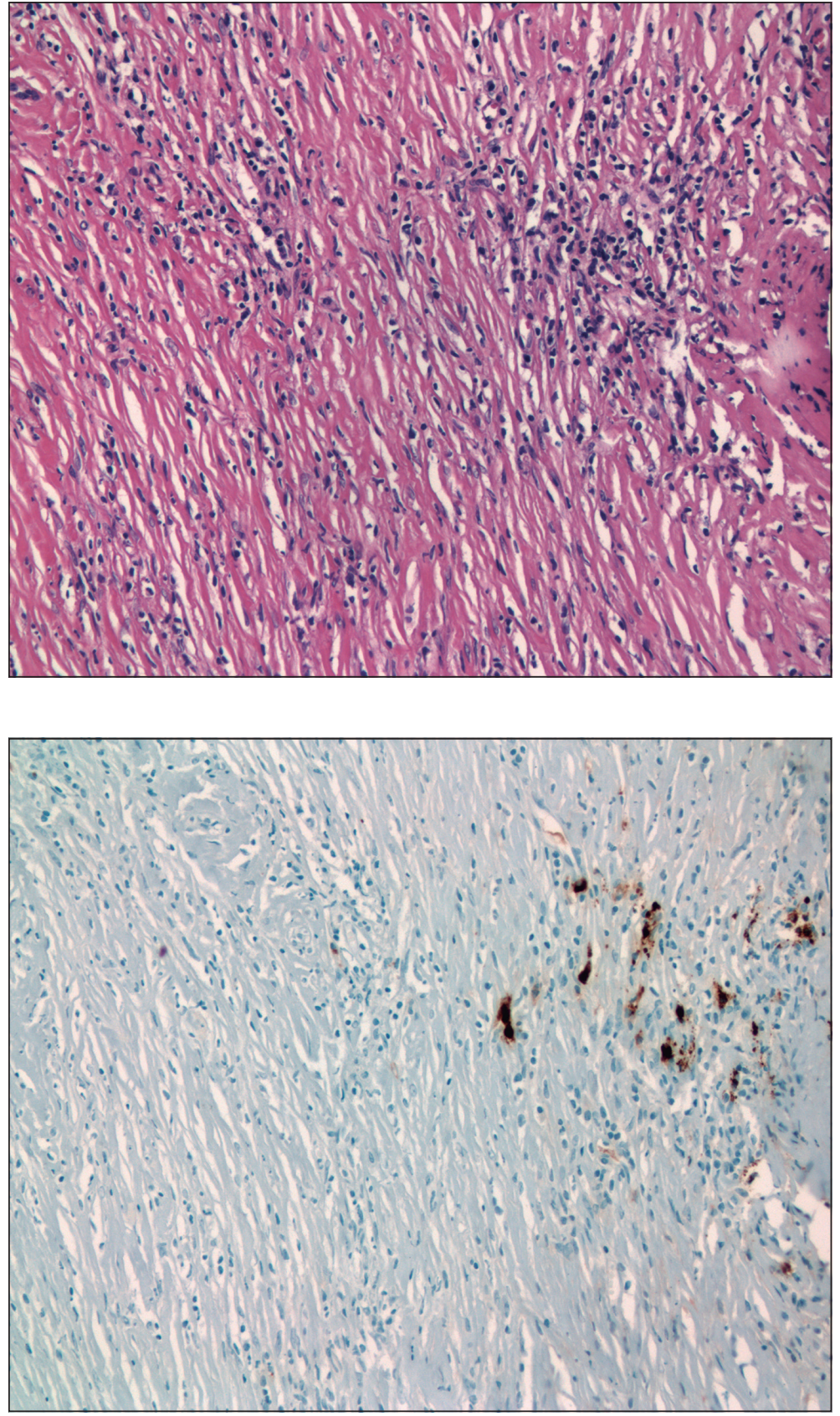

Figura 4. Tejido retroperitoneal con fibrosis densa con bandas colágenas paralelas gruesas, entre las cuales hay un aumento moderado de células inflamatorias (linfocitos, células plasmáticas) (Hematoxilina y eosina; 200x).

Figura 5. La misma área del retroperitoneo muestra focos de infiltrado de células plasmáticas con reactividad para lgG4 (Tinción inmunohistoquímica con acticuerpos monoclonales anti $\operatorname{lgG} 4$; 200x).

de colchicina c/12 h. Los síntomas desaparecieron rápidamente con normalización de la creatinina. La concentración plasmática de IgG4 (medida bajo terapia esteroidal) fue normal. La inmunohistoquímica del tejido retroperitoneal mostró células plasmáticas productoras de IgG4 en el tejido fibroso (Figuras 4 y 5). Cuatro meses después los catéteres fueron removidos. En los años siguientes el tejido retroperitoneal y perirrenal desapareció.

Veinticinco años después de su primera evaluación, la paciente no tiene signos radiológicos de hipofisitis ni FRP, mantiene la suplementación hormonal y tiene un control adecuado de sus otras patologías crónicas. 


\section{Discusión}

La disfunción hipotálamo-hipofisaria de esta paciente es llamativa por la presencia de DI, deficiencia gonadotropa, tirotropa y corticotropa, así como hiperprolactinemia y trastornos en el campo visual debido a extensión supraselar de la infiltración linfoplasmocitaria de la hipofisitis.

Las hipofisitis son un grupo heterogéneo de condiciones en que el subtipo linfocítico es el más frecuente. Afecta a mujeres jóvenes en edad reproductiva y frecuentemente postparto ${ }^{1,3}$. La hipofisitis linfoplasmocitaria es de las menos frecuentes y se asocia a IgG4-RD. En esta paciente se manifestó clínicamente por diabetes insípida a los 27 años y radiológicamente a los 35 . El eje corticotropo se afectó antes del eje tirotropo, lo cual es frecuente en las enfermedades inflamatorias e infiltrativas y difiere de los macroadenomas ${ }^{1}$. Frecuentemente la hipofisitis se asocia a otras condiciones autoinmunes, con múltiples autoanticuerpos contra enzimas y hormonas, y pueden ser parte del espectro de las manifestaciones de APS ${ }^{8-10,12,13}$. Estas condiciones autoinmunes pueden preceder o suceder al compromiso pituitario -usualmente linfocítico y no linfoplasmocitario como en esta paciente-siendo la tiroiditis de Hashimoto la más común, pudiendo coexistir con diabetes mellitus 1, insuficiencia adrenal, anemia perniciosa, vitíligo, hipoparatiroidismo, enfermedad celiaca, LES y artritis reumatoide ${ }^{3}$. Esta paciente se presentó con manifestaciones de APS que pueden clasificarse en el tipo 3 debido a la ausencia clínica de adrenalitis autoinmune.

Años después de las manifestaciones de hipofisitis linfoplasmocitaria y APS tipo 3, la paciente presentó una FRP. El uso de cabergolina podría ser invocado como su causa; sin embargo, el corto tiempo de uso y el tiempo entre éste y la aparición de la FRP lo hacen improbable ${ }^{15}$.

Otra explicación para la asociación de hipofisitis y FRP es la ocurrencia de IgG4-RD. Esta condición se caracteriza por inflamación, infiltración por células plasmáticas productoras de IgG4 y fibrosis en páncreas, pituitaria, glándulas salivales y lagrimales, tiroides, retroperitoneo, mesenterio, pericardio, meninges, órbitas, vía biliar, pulmones, riñones, piel, senos paranasales e hígado. Estos pacientes son predominantemente hombres, frecuentemente con niveles elevados de IgG4 en plasma que mejoran con el uso de esteroides $^{16,17}$. Aunque esta paciente tuvo niveles plasmáticos normales de IgG4 -probablemente por el uso previo de corticoides o por efecto prozona o hook- el análisis tisular de hipófisis y tejido retroperitoneal mostró infiltración por células plasmáticas productoras de IgG4, lo que constituye el fundamento diagnóstico de IgG4$\mathrm{RD}$. La respuesta de la hipofisitis a los corticoides no fue la esperada, requiriendo de una segunda resección y radioterapia para poder controlar el trastorno del campo visual.

El diagnóstico de IgG4-RD requiere la presencia de varios criterios establecidos por Leporati et cols. Esta paciente presentó 3 de 5 de ellos: infiltración por células IgG4 (+), masa selar en la RM y compromiso de otros órganos comprobado por biopsia².

El tratamiento de la FRP son los corticoides. Si la respuesta no es óptima o para reducir las dosis de esteroides, algunos grupos han utilizado otras drogas inmunomoduladoras como ciclofosfamida, azatioprina, micofenolato, ciclosporina, tamoxifeno y colchicina ${ }^{18-24}$. En este caso la prednisona y colchicina lograron controlar la enfermedad.

En conclusión, la hipofisitis linfoplasmocitaria es una condición infrecuente que puede presentarse en pacientes con IgG4-RD y asociarse a FRP. $\mathrm{Su}$ asociación con APS no ha sido comunicada previamente, pudiendo tener estos trastornos una patogenia común.

\section{Referencias}

1. Fukuoka H. Hypophysitis. Endocrinology and metabolism Clinics of North America 2015; 44 (1): 143-9.

2. Leporati P, Landek-Salgado MA, Lupi I, Chiovato L, Caturegli P. IgG4-related hypophysitis: a new addition to the hypophysitis spectrum. The Journal of clinical endocrinology and metabolism 2011; 96 (7): 1971-80.

3. Rivera JA. Lymphocytic hypophysitis: disease spectrum and approach to diagnosis and therapy. Pituitary 2006; 9 (1): 35-45.

4. Ray DK, Yen CP, Vance ML, Laws ER, Lopes B, Sheehan JP. Gamma knife surgery for lymphocytic hypophysitis. Journal of neurosurgery 2010; 112 (1): 118-21.

5. Bando H, Iguchi G, Fukuoka H, Taniguchi M, Yamamoto M, Matsumoto R, et al. The prevalence of IgG4-related hypophysitis in 170 consecutive patients with hypopituitarism and/or central diabetes insipidus 
and review of the literature. European journal of endocrinology/European Federation of Endocrine Societies 2014; 170 (2): 161-72.

6. Scheel PJ Jr, Feeley N. Retroperitoneal fibrosis. Rheumatic diseases clinics of North America 2013; 39 (2): 365-81.

7. Pipitone N, Vaglio A, Salvarani C. Retroperitoneal fibrosis. Best practice \& research Clinical rheumatology 2012; 26 (4): 439-48.

8. Schatz DA, Winter WE. Autoimmune polyglandular syndrome. II: Clinical syndrome and treatment. Endocrinology and metabolism clinics of North America 2002; 31 (2): 339-52.

9. Eisenbarth GS, Gottlieb PA. Autoimmune polyendocrine syndromes. The New England journal of medicine 2004; 350 (20): 2068-79.

10. Betterle C, Dal Pra C, Mantero F, Zanchetta R. Autoimmune adrenal insufficiency and autoimmune polyendocrine syndromes: autoantibodies, autoantigens, and their applicability in diagnosis and disease prediction. Endocrine reviews 2002; 23 (3): 327-64.

11. Devoto E, Aravena L, Corvalán L, Lioi X, Munizaga F, Soto N, et al. Autoimmune polyglandular syndrome. Clinical experience in 21 cases. Rev Med Chile 1990; 118 (6): 635-42.

12. Abramson J, Husebye ES. Autoimmune regulator and self-tolerance - molecular and clinical aspects. Immunological reviews 2016; 271 (1): 127-40.

13. Cutolo M. Autoimmune polyendocrine syndromes. Autoimmunity reviews 2014; 13 (2): 85-9.

14. Hamano H, Kawa S, Horiuchi A, Unno H, Furuya N, Akamatsu T, et al. High serum IgG4 concentrations in patients with sclerosing pancreatitis. The New England journal of medicine 2001; 344 (10): 732-8.

15. Lafeber M, Stades AM, Valk GD, Cramer MJ, Teding van Berkhout F, Zelissen PM. Absence of major fibrotic adverse events in hyperprolactinemic patients treated with cabergoline. European journal of endocrinology/
European Federation of Endocrine Societies 2010; 162 (4): 667-75.

16. Nambam B, Winter WE, Schatz DA. IgG4 antibodies in autoimmune polyglandular disease and IgG4-related endocrinopathies: pathophysiology and clinical characteristics. Current opinion in pediatrics 2014; 26 (4): 493-9.

17. Kawano M, Saeki T, Nakashima H, Nishi S, Yamaguchi $\mathrm{Y}$, Hisano S, et al. Proposal for diagnostic criteria for IgG4-related kidney disease. Clinical and experimental nephrology 2011; 15 (5): 615-26.

18. Swartz RD. Idiopathic retroperitoneal fibrosis: a review of the pathogenesis 266 and approaches to treatment. American journal of kidney diseases 2009; 54 (3): 54653.

19. Vega J, Goecke H, Santamarina M. Retroperitoneal fibrosis associated with chronic use of ergotamine: report of one case. Rev Med Chile 2011; 139 (4): 489-94.

20. Vega J, Ceriani A, Jensen D. Retroperitoneal fibrosis associated with malignancies: report of eight cases. Rev Med Chile 2014; 142 (6): 791-8.

21. Vega J, Goecke H, Tapia H, Labarca E, Santamarina M, Martínez G. Treatment of idiopathic retroperitoneal fibrosis with colchicine and steroids: a case series. American journal of kidney diseases 2009; 53 (4): 628-37.

22. Cristian S, Cristian M, Cristian P, Constantin G, Savu C, Huri E, et al. Management of idiopathic retroperitoneal fibrosis from the urologist's perspective. Therapeutic advances in urology 2015; 7 (2): 85-99.

23. Vega J, González J, Goecke H, Carrasco A, Santamarina M. Fibrosis retroperitoneal idiopática metacrónicamente asociada a un seudotumor de cabeza de páncreas. Terapia exitosa con corticoides y colchicina. NefroPlus 2011; 4: 39-44.

24. Joshi MN, Whitelaw BC, Carroll PV. Mangement of endocrine disease: Hypophysitis-diagnosis and treatment. Eur J Endocrinol 2018; Jun 7, pii. EJE-17-0009 (Epub ahead of print). 